Revue d'histoire de l'Amérique française

BRS REVUE D.HISTOIRE DE L'AMÉRIQUE FRANÇAISE

\title{
L’Institut Canadien de Montréal et l'Institut National
}

\section{Léon Pouliot}

Volume 14, numéro 4, mars 1961

URI : https://id.erudit.org/iderudit/302078ar

DOI : https://doi.org/10.7202/302078ar

Aller au sommaire du numéro

Éditeur(s)

Institut d'histoire de l'Amérique française

ISSN

0035-2357 (imprimé)

1492-1383 (numérique)

Découvrir la revue

Citer cet article

Pouliot, L. (1961). L'Institut Canadien de Montréal et l'Institut National. Revue d'histoire de l'Amérique française, 14(4), 481-486.

https://doi.org/10.7202/302078ar d'utilisation que vous pouvez consulter en ligne.

https://apropos.erudit.org/fr/usagers/politique-dutilisation/ 


\section{L'INSTITUT CANADIEN DE MONTRÉAL ET L'INSTITUT NATIONAL}

Après avoir raconté la crise que connut l'Institut Canadien de Montréal en 1858, comme conséquence de l'intervention de Mgr Bourget, le P. Théophile Hudon écrit: «Labrèche-Viger et Édouard Fabre, chefs de la scission, fondèrent avec leurs amis l'Institut National; celui-ci s'éteignit quelques années après, non sans avoir accompli un bon travail. ${ }^{1}$

Il y a là une inexactitude qu'il nous paraît opportun de relever, d'autant qu'elle a reçu une large publicité dans le bel ouvrage de M. Mason Wade: The French Canadians. ${ }^{2}$ Le P. Hudon pensait, croyons-nous, à l'Institut Canadien-Français, fondé le 3 mai $1858^{3}$ et qui s'était assigné comme tâche de circonscrire l'influence de l'Institut Canadien tout court. Quant à l'Institut National, il est antérieur de six ans aux Pastorales de Mgr Bourget et à la crise de 1858. Comment a-t-il vu le jour et quel but poursuivaient ses fondateurs?

A notre avis, ce n'est pas dans les règlements écrits de l'Institut National qu'il faut en chercher la raison profonde, mais dans les circonstances lointaines et prochaines qui ont présidé à ses origines. Les élections parlementaires de 1848 avaient été une première épreuve de force pour l'Institut Canadien. Ses membres n'étaient pas restés indifférents à la mêlée; ils étaient descendus dans l'arène électorale, les uns soutenant Papineau, les autres LaFontaine, avec toute la violence caractéristique de l'époque. La belle harmonie qui avait régné jusque-là était rompue. On le vit bien quand l'Institut renouvela son

${ }^{1}$ L'Institut Canadien et l'affaire Guibord (Montréal, Beauchemin, 1938), 59.

2 (Toronto, MacMillan, 1955), 344: « The dissidents, supported by the Bishop, opened the short-lived Institut National. 》

3 Huguet-Latour, Annuaire de Ville-Marie (Montréal, 1864), I: 184. E.-Z. Massicotte fixe au 14 mai la date de la fondation, BRH (1941), 238. 
conseil; il dut s'y reprendre à deux fois avant de réussir, chaque parti luttant désespérément pour le dominer et par là l'annexer à sa politique. Le parti de Papineau l'emporta.

Malgré des défections, l'Institut continua son chemin; il progressa, il reconstitua sa bibliothèque détruite par l'incendie de 1850. Il était toujours la seule société littéraire de langue française à Montréal, la seule qui offrit au public des conférences dont celui-ci était alors si friand. Et voilà qu'il rêve d'étendre et de solidifier son emprise sur la ville. Que veut-il ? Un immeuble qui abritera tous ses services et dont il sera le seul propriétaire. ${ }^{4}$ Mais il est toujours dominé par le parti de Papineau; ses membres siègent à la rédaction de l'Avenir. Et l'Avenir, mal vu du parti de LaFontaine, se donne encore le tort de heurter de front le sentiment catholique. La Minerve du 2 décembre 1850 publie une lettre, signée Pierre, et dont voici le contenu: «L'Institut Canadien blâme les Montréalais de ne pas le soutenir. Il est anti-clérical, anti-catholique; il s'est réjoui de voir Pie IX exilé à Gaète, il regrette de le voir revenu à Rome. Que l'Institut cesse de nous mépriser avant de solliciter notre appui. » Des membres de l'Institut sont en pleine sympathie avec Pierre; et c'est pourquoi au début de 1851, ils tentent, mais en vain, de bannir L'Avenir de la salle de lecture. A la réunion du 12 juin 1851, Magloire Desnoyers présente une série de résolutions qui ont pour but d'interdire les discussions politiques au sein de l'Institut. Un amendement est aussitôt proposé: "Qu'il soit résolu que, aujourd'hui comme par le passé, l'Institut Canadien de Montréal ne soutient comme corps aucun parti politique. » Et l'amendement est adopté par 76 voix contre $36 .^{5}$ C'est cet échec, croyonsnous, qui est à l'origine de l'Institut National. On travailla d'abord dans l'ombre. La Minerve du 23 avril 1852 fait écho à une rumeur selon laquelle on s'efforce sans bruit de ruiner l'Institut: la direction en est émue, elle crie à la persécution, elle a peur. Et pour empêcher le désastre, elle consentirait volontiers

${ }^{4}$ La Minerve, 16 avril 1852. Rapport du comité nommé par l'Institut Canadien pour étudier ce problème.

5 Hector Berthelot, Le bon vieux temps (Montréal, Beauchemin, 1924), 99. Aussi Massicotte, dans BRH (1941), 236. 
à se donner comme président et vice-président des membres aux idées modérées, moins compromis devant l'opinion publique. ${ }^{6}$

La rumeur n'était pas sans fondement. Une lettre insérée dans La Minerve du 10 novembre 1852 nous apprend qu'il était question depuis le mois de février de fonder une nouvelle société littéraire à Montréal. Quel nom lui donner ? On songea d'abord à l'Institut Catholique. Mgr Bourget pressenti, approuva; mais cet Institut, ajoutait-il, devrait se recruter chez les Irlandais comme chez les Canadiens français. ${ }^{7}$ La suggestion ne fut pas agréée, et pour cause. Comme il existait déjà un Institut Catholique Irlandais, ${ }^{8}$ le nouveau venu risquait de ne pas avoir ses coudées franches, sans parler des autres inconvénients inhérents à l'organisation et au fonctionnement d'une société littéraire bilingue et bi-ethnique. Mais surtout, si, comme tout nous porte à le croire, on voulait, sous le couvert d'une société littéraire, miner l'influence politique de l'Institut Canadien, les Irlandais n'avaient rien à faire dans cette galère.

On se ravisa donc, et le 17 avril 1852, Mgr Bourget acceptait d'être le patron de l'Institut National. Il disait tout le bien que peuvent faire des laïques instruits et louait la décision de ne garder dans la bibliothèque dudit Institut que des bons livres. ${ }^{9}$ L'heure de l'action a sonné; croyons-en La Minerve du 22 mai :

Institut National. Nous avons le plaisir d'annoncer à nos lecteurs qu'il s'est formé dans cette ville une nouvelle société littéraire dont le but est de procurer à la jeunesse de toutes les classes un moyen facile de puiser des connaissances utiles. L'un des objets louables que cette association a pour but de réaliser est d'ouvrir une chambre de nouvelles que les marchands canadiens pourront fréquenter avec avantage

6 Lettre signée: $O n$-dit.

${ }^{7}$ AAM. Lettres de Mgr Bourget, 7: 273-274, à M. J. - L. Beaudry, 14 avril 1852.

${ }^{8}$ Le célèbre converti américain, Brownson, donnait, en ce moment même des conférences à Montréal sous les auspices de l'Institut Catholique Irlandais.

9 AAM. Lettres de Mgr Bourget, 7: 275-276, à MM. U. Baudry, Ouimet et Jodoin. On lit, dans la notice nécrologique du juge Baudry: "Il travailla avec zèle à la fondation de l'Institut National, dont il rédigea la constitution." L'Opinion Publique, 20 janvier 1876. 
et une bibliothèque d'ouvrages choisis et recommandables sous tous les rapports.

Plusieurs amis réunis un soir, vers le début du mois dernier, prirent la détermination de fonder cette société et de faire appel au patriotisme et à la générosité des citoyens, et nous devons le dire, l'expérience a prouvé qu'ils ne pouvaient mieux s'adresser, comme nous le ferons voir bientôt, en donnant, dans notre prochain numéro probablement, un rapport détaillé de l'état de la société, et en publiant les règlements et les noms des officiers. Il suffira de dire, pour le moment, que J.-L. Beaudry, écr. a été porté à la présidence par le choix judicieux des membres.

Comme on le voit, l'Institut National poursuit le même but que l'Institut Canadien; répandre les connaissances utiles, éclairer le peuple. Et il emploie les mêmes moyens: salle de lecture, bibliothèque, conférences publiques. Il offre en plus une double garantie: patronage de l'évêque et orthodoxie de la bibliothèque. Il espérait par là rallier les catholiques cultivés, amis du progrès intellectuel, mais qui éprouvaient un véritable malaise devant les idées trop avancées, voire impies, affichées ouvertement par les chefs de l'Institut Canadien.

La première conférence publique de l'Institut National eut lieu le 26 mai 1852. Après le discours d'ouverture de Toussaint Pelletier, T.-J.-J. Loranger répondit à la question suivante: * Quels sont les avantages et les désavantages du roman comme genre littéraire ?» Et ce fut un beau début: «La salle était remplie et la réunion comptait surtout une très forte proportion de citoyens d'une haute respectabilité, de position et d'influence dans la société montréalaise. ${ }^{10}$ Cependant on ne signale pas la présence de Mgr Bourget. Le sujet traité par Loranger n'était pas de nature à intéresser l'évêque de Montréal, même des laïcs sérieux de l'époque ayant peu d'estime pour le roman. ${ }^{11}$ Mais comme c'était la première manifestation de l'Institut National, l'absence de son Patron n'est pas sans nous inquiéter un peu.

10 La Minerve, 29 mai 1852.

11 Denis-Benjamin Viger, décédé en 1861, se félicitait, dans sa dernière maladie, de n'avoir jamais lu de romans. 
Peut-être s'était-il rendu compte que la nouvelle société littéraire poursuivait des fins politiques.

$\mathrm{Au}$ début, les deux Instituts coexistent pacifiquement, sinon amicalement. On pouvait appartenir à l'un et à l'autre. C'est le cas de l'avocat Tailhades, décédé en novembre 1852 ; membrefondateur de l'Institut National, il n'avait pas cessé d'appartenir à l'Institut Canadien. A la même date, M. Ossaye, second conférencier de l'Institut National est toujours membre de l'Institut Canadien.

Il ne devait pas en être toujours ainsi. Au printemps de 1854, l'Institut Canadien voyait s'achever l'édifice qui abriterait tous ses services. Espéra-t-il, à cette occasion, absorber l'Institut National? On serait porté à le croire en lisant La Minerve du 8 avril:

On nous informe que des adversaires de l'Institut National ont fait courir le bruit que cette institution allait s'éteindre pour se fondre avec l'Institut Canadien. Nous pouvons informer le public qu'il n'en sera pas ainsi et que les membres de cet Institut ne transigeront pas avec leurs principes jusqu'au point de délibérer sur la question de savoir s'il leur sera défendu de proscrire les mauvais livres de leur bibliothèque.

La Minerve, journal ministériel, se portant à la défense de l'Institut National et à l'attaque de l'Institut Canadien, en fautil davantage pour nous convaincre que, sous prétexte de littérature, l'Institut National servait, lui aussi, des fins politiques ? Il y a mieux encore:

On nous demande pourquoi la Minerve n'a rien dit de l'inauguration des nouvelles salles de l'Institut Canadien. Nous répondons que nous n'avons pas l'habitude de nous occuper des affaires de cette institution, que nous n'étions pas présent, et que personne de ceux qui y étaient n'a jugé à propos de nous en écrire un mot. Les autres journaux disent que Mr Fleury St-Jean et M. Galarati ont bien chanté ce soir-là, que les orateurs ont parlé comme des Démosthènes, et que la salle était trop petite pour contenir la foule qui voulait y entrer. ${ }^{12}$

\footnotetext{
${ }^{12}$ La Minerve, 9 mai 1854.
} 
La mauvaise humeur du rédacteur est manifeste. Mais quel en est le mobile ? Littéraire ou politique ?

Notre propos n'étant pas de raconter l'histoire de l'Institut National, arrêtons-nous ici. Nous en avons dit assez pour établir qu'il est antérieur à la crise de 1858. Combien de temps a-t-il vécu ? D'après E.-Z. Massicotte, «il n'aurait fait que vivoter jusqu'en $1855 »^{13}$

LÉON POULIOT, S.J.

13 BRH (1941), 238.

Articles à paraître :

Nous avons voulu mettre fin, en cette livraison de la Revue, à des études sectionnées déjà depuis longtemps. Donc à paraître aussitôt que possible:

Gaston Carrière, o.m.i.: Projets de préfectures apostoliques pour le nord-est du Canada.

Jeanne Grégoire: Les Acadiens dans une seconde patrie: la Louisiane.

Jeanne Grégoire: Louisiane et langue française.

L. Jore: Un Canadien, gouverneur du Sénégal, Louis Le Gardeur de Repentigny, 1721-1786.

Roland Lamontagne: La construction du fort Saint-Jean, 174817.49 .

Robert Le Blant: Notes sur Jean de Garibal, associé de NotreDame-de-Montréal, vers le 24 juillet $1619-17$ juillet $166 \%$.

Jean Côté, s.j.: L'Institution des donnés à Sainte-Marie-desHurons.

Etc. 